\title{
On the Solutions of the Rational Covariance Extension Problem Corresponding to Pseudopolynomials Having Boundary Zeros
}

\author{
Hendra I. Nurdin and Arunabha Bagchi
}

\begin{abstract}
In this paper, we study the rational covariance extension problem when the chosen pseudopolynomial of degree at most $n$ has zeros on the boundary of the unit circle. In particular, we derive a necessary and sufficient condition for a solution to be bounded (i.e. has no poles on the unit circle). Furthermore, we propose a new procedure for computing all bounded solutions for this special case of zeros of pseudopolynomials on the boundary and illustrate it by means of two examples.
\end{abstract}

\section{INTRODUCTION}

Recent years have seen significant advances in the theory of analytic interpolation on the open unit disc of the complex plane. Some major results are the parametrization of all positive real rational functions interpolating a certain positive partial covariance sequence $c_{o}, c_{1}, \ldots, c_{n}$, in terms of desirable "spectral zeros" and the introduction of a convex optimization based approach to compute the solution [1], [2], [3], [4], [5]. However, that particular approach is only theoretically known to be applicable to the case where none of the spectral zeros lie on the unit circle. The remaining case where there are spectral zeros on the unit circle is important not only for the sake of completeness, but also due to the fact that placing or forcing a zero on the unit circle is desirable in the design of some filters. In this paper, we derive theoretical results for this special case based on the convex optimization approach. In particular, we illustrate how to arrive at a bounded solution by working out two examples, and propose a general technique for approaching the problem. For an alternative approach to the problem, see [6]. However, our method gives new results which provide additional insight into the behavior of the solutions.

The organization of this paper is as follows: In Section II we introduce the notation and basic definitions that are used throughout the paper. In Section III we recall relevant results from the literature. In Section IV we use a convex optimization approach to derive new results on the rational covariance extension problem when there are spectral zeros on the unit circle. Based on those results, we introduce a computational procedure for obtaining bounded solutions. Finally, in Section V we offer some concluding remarks.

The authors are with the Systems, Signals and Control Group, Department of Applied Mathematics, University of Twente, P.O Box 217, 7500 AE Enschede, The Netherlands E-mail: $\{$ h.i.nurdin, a.bagchi\}@math. utwente.nl

\section{Notation AND Definitions}

- $\bar{A}, \operatorname{int}(A), \partial A, A^{c}$ denotes the closure, open interior, boundary, and complement of a set $A$, respectively.

- $\mathbb{R}, \mathbb{C}$, and $\mathbb{D}$ denote the set of real numbers, complex numbers, and the open unit disc $=\{z \in \mathbb{C}:|z|<1\}$, respectively.

- For any $z \in \mathbb{C}, \bar{z}$ denotes its complex conjugate.

- $\operatorname{col}\left(a_{1}, \ldots, a_{n}\right)$ denotes a column vector with elements $a_{1}, \ldots, a_{n}$.

- A complex function $f$ is said to be real if $f(z) \in \mathbb{R}$ for all $z \in \mathbb{R}$.

- $\mathcal{H}(\mathbb{D})$ denotes the set of functions holomorphic in $\mathbb{D}$.

- For any $f \in \mathcal{H}(\mathbb{D}), f_{*}$ is defined by $f_{*}(z)=\overline{f\left(\bar{z}^{-1}\right)}$.

- $\mathcal{H}^{\infty}(\mathbb{D})$ denotes the (Hardy) space of functions in $\mathcal{H}(\mathbb{D})$ which are bounded in $\mathbb{D}$.

- $\mathcal{C}$ denotes the Carathèodory class $=\{f \in \mathcal{H}(\mathbb{D})$ : $\operatorname{Re}\{f(z)\} \geq 0 \forall z \in \mathbb{D}\}$ and $\mathcal{C}_{+}$denotes the subset $\left\{f \in \mathcal{C}: \inf _{z \in \partial \mathbb{D}} \operatorname{Re}\{f(z)\}>0\right\}$ of $\mathcal{C}$.

- The degree of a proper rational function $f \in \mathcal{C}$ is its McMillan degree, i.e. the dimension of a minimal statespace realization of $f(z)$.

- By a pseudopolynomial we mean any complex function $f$ of the form $f(z)=\sum_{i=-m}^{n} a_{i} z^{i}$, where $0 \leq m, n<$ $\infty$, and $a_{i} \in \mathbb{C}$ for $i=-m,-m+1, \ldots, n$, and by a symmetric pseudopolynomial we mean a pseudopolynomial of the form

$$
f(z)=a_{0}+\sum_{i=1}^{n}\left(\overline{a_{i}} z^{-i}+a_{i} z^{i}\right)
$$

where $0 \leq n<\infty, a_{n} \neq 0$, and $\left(a_{0}, a_{1}, \ldots, a_{n}\right) \in$ $\mathbb{R} \times \mathbb{C}^{n}$. We say that $n$ is the order of the symmetric pseudopolynomial $f$ (the order is zero if $f$ is a constant function).

- A point $z \in \partial \mathbb{D}$ is said to be boundary zero for a symmetric pseudopolynomial $f$ if $f(z)=0$.

- For any set $A \subseteq \mathbb{C}, \mathcal{Q}(n, A)$ denotes the set of all symmetric pseudopolynomials of order $n$ with $\left(a_{0}, a_{1}, \ldots, a_{n}\right) \in \mathbb{R} \times A^{n}$.

- For any set $A \subseteq \mathbb{C}, \mathcal{Q}_{+}(n, A)$ denotes the set of all symmetric pseudopolynomials in $\mathcal{Q}(n, A)$ which are positive $(>0)$ on $\partial \mathbb{D}$, i.e. $f(z)>0 \forall z \in \partial \mathbb{D}$. On this set we associate the topology induced by the maximum norm:

$$
\|f\|_{\infty}=\max _{z \in \partial \mathbb{D}}|f(z)| \forall f \in \mathcal{Q}_{+}(n, A)
$$


- For any set $A \subseteq \mathbb{C}, \mathfrak{Q}_{+}(n, A)=\bigcup_{k=0}^{n} \mathcal{Q}_{+}(k, A)$. We also associate the topology induced by the $\|\cdot\|_{\infty}$ norm on this set.

Remark 1: One should take care not to confuse $\mathcal{Q}_{+}(n, A)$ with $\mathfrak{Q}_{+}(n, A)$.

\section{Mathematical Preliminaries}

\section{A. The Rational Interpolation and Rational Covariance} Extension Problem

Before formally defining the rational interpolation problem (RIP) and the rational covariance extension problem (RCEP) we state a definition:

Definition $2(n+1$-interpolation data $)$ : Let $\mathcal{Z}_{n+1}=$ $\left\{z_{k}, k=0,1, \ldots, n\right\}$ be an indexed set of $n+1$ points in $\mathbb{D}$ (not necessarily distinct) and let $\mathcal{W}_{n+1}=\left\{w_{k}, k=\right.$ $0,1, \ldots, n\}$ be an indexed set of $n+1$ points in $\mathbb{C}$. We always assume that the indexing of points in $\mathcal{Z}_{n+1}$ is such that non-distinct points are ordered consecutively. The pair $\left(\mathcal{Z}_{n+1}, \mathcal{W}_{n+1}\right)$ is said to be an $n+1$-interpolation data.

We now state the rational interpolation problem (RIP):

Problem 3 (RIP): Given an $n+1$-interpolation data $\left(\mathcal{Z}_{n+1}, \mathcal{W}_{n+1}\right)$, find all proper rational functions $f \in \mathcal{C}$ of degree less than or equal to $n$ such that:

$$
f\left(z_{k}\right)=w_{k}
$$

if $z_{k}$ has multiplicity 1 (i.e. $z_{k}$ only appears once in $\mathcal{Z}_{n+1}$ ) and

$$
\frac{1}{l !} f^{(l)}\left(z_{k+l}\right)=w_{k+l}, l=0,1, \ldots, m-1
$$

if $z_{k}=z_{k+1}=\ldots=z_{k+m-1}$.

It is known that the RIP has a solution if and only if the so-called (generalized) Pick matrix associated with the given $n+1$-interpolation data (for a definition, see, e.g., [7]), which is denoted by $\mathbf{P}\left(\mathcal{Z}_{n+1}, \mathcal{W}_{n+1}\right)$, is nonnegative definite. If the (generalized) Pick matrix is nonnegative definite but singular then there is a unique solution, while if it is positive definite then there is an infinite number of solutions. Since we are only interested in the second case, from this point onwards we shall always assume that $\mathbf{P}\left(\mathcal{Z}_{n+1}, \mathcal{W}_{n+1}\right)>0$ for the given $n+1$-interpolation data.

A special case of the RIP is the rational covariance extension problem (RCEP). The RIP becomes RCEP when $z_{0}=z_{1}=\ldots=z_{n}=0, w_{0}=\frac{1}{2} c_{0}$ and $w_{k}=c_{k}$ for $k=1,2, \ldots, n$. In the following we shall give a formal definition of the RCEP.

Definition 4: A sequence of complex numbers $c_{0}, c_{1}, \ldots, c_{n}$ (with $c_{0} \in \mathbb{R}$ ) is said to be a positive partial sequence (or more explicitly, a positive partial covariance sequence) if the Toeplitz matrix:

$$
\left[\begin{array}{ccccc}
c_{0} & \overline{c_{1}} & \overline{c_{2}} & \ldots & \overline{c_{n}} \\
c_{1} & c_{0} & \overline{c_{1}} & \ddots & \vdots \\
c_{2} & c_{1} & c_{0} & \ddots & \overline{c_{2}} \\
\vdots & \ddots & \ddots & \ddots & \overline{c_{1}} \\
c_{n} & \ldots & c_{2} & c_{1} & c_{0}
\end{array}\right]
$$

is positive definite.

Problem 5 (RCEP/Carathèodory problem): Given a positive partial sequence $c_{o}, c_{1}, \ldots, c_{n}(n \geq 1)$, find all proper rational functions $f \in \mathcal{C}$ of degree less than or equal to $n$ such that the first $n+1$ coefficients of the Taylor series expansion of $f$ about 0 is $\frac{1}{2} c_{o}, c_{1}, \ldots, c_{n}$.

\section{B. Results on the RIP, RCEP and Generalized Interpolation} on $\mathcal{H}^{\infty}(\mathbb{D})$

In a series of papers [1][2][4], it was finally established that given an $n+1$-interpolation data, one may associate to each pseudopolynomial $d \in \overline{\mathfrak{Q}_{+}(n, \mathbb{C})}$ a unique pair $(\pi, \chi)$ of polynomials of degree $\leq n$ depending only on the data and $d$ such that $f(\cdot)=\frac{\pi(\cdot)}{\chi(\cdot)}$ satisfies the requirements of the RIP. We state a pertinent result:

Theorem 6 ([4, Theorem 2]): For a given $n+1$ interpolation data $\left(\mathcal{Z}_{n+1}, \mathcal{W}_{n+1}\right)$ and any polynomial $\eta \neq 0$ of degree $\leq n$ with roots in $\mathbb{D}^{c}$ and normalized by $\eta(0)=1$, there is a unique pair of polynomials $(\pi, \chi)$ of degree $\leq n$ such that $\pi+\chi$ has all its roots in $\mathbb{D}^{c}$, the pair satisfies the relation

$$
\pi \chi_{*}+\chi \pi_{*}=\kappa^{2} \eta \eta_{*}
$$

for a fixed choice of $\kappa>0$, and $f=\frac{\pi}{\chi}$ satisfies the requirements of the RIP. Furthermore, any root of $\pi+\chi$ on $\partial \mathbb{D}$ is common to all three polynomials $\pi, \chi$ and $\eta$, in which case $f$ is an interpolating function of degree $<n$.

Thus we may parametrize all solutions of the RIP in terms of the set of polynomials $\eta$ (with roots in $\mathbb{D}^{c}$ and normalized at zero) and a constant $\kappa>0$, or, equivalently, in terms of elements $d \in \overline{\mathfrak{Q}_{+}(n, \mathbb{C})}$ where $d=\kappa^{2} \eta \eta_{*}$. We are now in a position to formulate the following two more specific problems:

1) The particular rational interpolation problem (PRIP).

2) The particular rational covariance extension problem (PRCEP).

Problem 7 (PRIP): Given an $n+1$-interpolation data $\left(\mathcal{Z}_{n+1}, \mathcal{W}_{n+1}\right)$ and a symmetric pseudopolynomial $\Psi \in$ $\overline{\mathfrak{Q}_{+}(n, \mathbb{C})} \backslash\{0\}$, find the proper rational function $f=\frac{a}{b} \in$ $\mathcal{C}$ of degree $\leq n$ such that:

1) If $z_{k}$ has multiplicity 1 (i.e. $z_{k}$ only appears once in $\left.\mathcal{Z}_{n+1}\right)$ then

$$
f\left(z_{k}\right)=w_{k}
$$

and if $z_{k}=z_{k+1}=\ldots=z_{k+m-1}$ then

$$
\frac{1}{l !} f^{(l)}\left(z_{k+l}\right)=w_{k+l}, l=0,1, \ldots, m-1
$$

2) $a b_{*}+b a_{*}=\Psi$.

Problem 8 (PRCEP): Given a positive partial sequence $c_{o}, c_{1}, \ldots, c_{n}(n \geq 1)$ and a pseudopolynomial $\Psi \in$

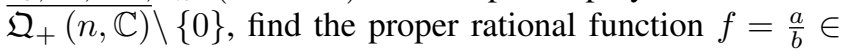
$\mathcal{C}$ of degree $\leq n$ such that the first $n+1$ coefficients of the Taylor series expansion of $f$ about 0 is $\frac{1}{2} c_{o}, c_{1}, \ldots, c_{n}$ and $a b_{*}+b a_{*}=\Psi$. 
Remark 9: Although we have treated both the RIP and RCEP, our concern in this paper is the RCEP. The discussion on the PRIP has been provided for completeness.

Remark 10: In practice one usually only deals with real polynomials and real pseudopolynomials. However, to ensure that $(\pi, \chi)$ will be real polynomials when $d$ is real, an additional constraint has to be imposed on the interpolation data. In the case of the PRCEP, the constraint is that $c_{0}, c_{1}, \ldots, c_{n}$ is a real sequence.

A convex optimization based method to compute the solution of the PRCEP for any given real valued positive partial sequence $c_{0}, c_{1}, \ldots, c_{n}$ and symmetric pseudopolynomial $\Psi \in \mathfrak{Q}_{+}(n, \mathbb{R})$ (i.e. $\Psi$ cannot have roots on $\partial \mathbb{D}$ ) was first introduced in [3], reappeared in [5], and was adapted to solve the Nevanlinna-Pick interpolation problem with degree constraint in [8]. Recently, ideas from [3], [5], [8] were generalized to a general setting of interpolation on $\mathcal{H}^{\infty}(\mathbb{D})$ with a so-called complexity constraint [9] which includes the PRIP and PRCEP as special cases.

However, one specialized aspect of the theory which has received relatively less attention is the case of solving the PRCEP when the given symmetric pseudopolynomial has zeros on the boundary (a recent work which does address it is [6]). In this paper we treat this special case by utilizing convex optimization, giving additional insight into the behavior of the solutions.

\section{Complete ReAl-VAlued, BOUNDED SOLUTiOnS OF THE PRCEP}

As we had mentioned in the last section, a method to compute the solution of the PRCEP for $\Psi \in \mathfrak{Q}_{+}(n, \mathbb{R})$ has been given in [3], [5]. However, looking back at the particular approach that was taken, it seems very plausible to extend it to the case where the prescribed symmetric pseudopolynomial has boundary zeros, i.e. $\Psi \in$ $\partial \mathfrak{Q}_{+}(n, \mathbb{R}) \backslash\{0\}$. By a more detailed analysis, we show in the following that this is indeed the case when the solution is bounded. By bounded, we mean that the solution does not have a pole on the unit circle. Then we illustrate how all bounded solutions can be computed. However, due to space limitation most proofs have been omitted. The complete proofs can be found in [10] (also serving as a revised version of [11]).

Notation 11: $\mathcal{C}_{+}^{\mathbb{R}}$ denotes the set of real functions in $\mathcal{C}_{+}$.

The set $\mathcal{C}_{+}^{\mathbb{R}}$ has been introduced because we are only interested in real solutions of the RCEP. Therefore, we always take the given positive partial sequence and symmetric pseudopolynomials to be real.

Definition 12: $\Phi(f)=f+f_{*} \forall f \in \mathcal{H}(\mathbb{D})$.

Definition 13: The mapping $Q: \mathbb{R}^{n+1} \rightarrow \bigcup_{k=0}^{n} \mathcal{Q}(k, \mathbb{R})$ is defined by:

$$
Q\left(q_{0}, q_{1}, q_{2}, \ldots, q_{n}\right)(z)=q_{0}+\sum_{i=1}^{n} \frac{1}{2} q_{i}\left(z^{-i}+z^{i}\right)
$$

Remark 14: Clearly $Q$ is a bijection.
Remark 15: Since it will often be used, one should make note of the mapping $Q$ that has been defined in (4).

Definition 16: Let $X$ be a convex set and let $F: X \rightarrow$ $\mathbb{R} \cup\{-\infty\}$ be a concave functional on $X$. Then the effective domain of $F$, denoted by $D_{e}(F)$, is defined as:

$$
D_{e}(F)=\{x \in X: F(x)>-\infty\}
$$

where $D_{e}(F)$ is again a convex set.

Remark 17: Maximizing a concave functional $F: X \rightarrow$ $\mathbb{R} \cup\{-\infty\}$ over $X$ is equivalent to maximizing $\left.F\right|_{D_{e}(F)}$ : $D_{e}(F) \rightarrow \mathbb{R}$ over $D_{e}(F)$ (see [12]).

Remark 18: For shorthand we shall often write $\int_{-\pi}^{\pi} f\left(e^{i \theta}\right) g\left(e^{i \theta}\right) d \theta$ as $\int_{-\pi}^{\pi} f g$

In our approach, we will consider the set $\mathcal{C}_{+}^{\mathbb{R}} \cap \mathcal{H}^{\infty}(\mathbb{D})$, following the setting of [9], and its closure $\overline{\mathcal{C}_{+}^{\mathbb{R}} \cap \mathcal{H}^{\infty}(\mathbb{D})}$. This goes in line with our interest in studying bounded solutions of the RCEP.

Lemma 19: $\mathcal{C}_{+}^{\mathbb{R}} \cap \mathcal{H}^{\infty}(\mathbb{D})$ is a convex set while $\overline{\mathcal{C}_{+}^{\mathbb{R}} \cap \mathcal{H}^{\infty}(\mathbb{D})}$ is a closed, convex set.

Definition 20: Given a linear space $X$ and a subset $Y$ of $X$, an element $g \in X$ is said to be a feasible direction relative to $Y$ for an element $f \in Y$ if $\exists \sigma>0$ such that $f+h g \in Y$ for all $0<h \leq \sigma$.

Definition 21: The functional $\mathbb{I}_{\Psi}: \overline{\mathcal{C}_{+}^{\mathbb{R}} \cap \mathcal{H}^{\infty}(\mathbb{D})} \rightarrow$ $\mathbb{R} \cup\{-\infty\}$ is defined as:

$$
\mathbb{I}_{\Psi}(f)=\frac{1}{2 \pi} \int_{-\pi}^{\pi} \Psi\left(e^{i \theta}\right) \log \Phi(f)\left(e^{i \theta}\right) d \theta
$$

Following [3], [5], we wish to maximize $\mathbb{I}_{\Psi}$ over all functions $f$ in $\overline{\mathcal{C}_{+}^{\mathbb{R}} \cap \mathcal{H}^{\infty}(\mathbb{D})}$ satisfying the interpolation constraints

$$
\frac{1}{2 \pi} \int_{-\pi}^{\pi} \Phi(f) e^{i k \cdot}=c_{k} \text { for } k=0,1, \ldots, n .
$$

However, in this paper we allow $\Psi$ to have boundary zeros (i.e. $\Psi$ can be in $\left.\partial \mathfrak{Q}_{+}(n, \mathbb{R}) \backslash\{0\}\right)$. First, we state a useful lemma:

Lemma 22: For any $\Psi \in \overline{\mathfrak{Q}_{+}(n, \mathbb{R})} \backslash\{0\}$, the functional $\mathbb{I}_{\Psi}$ is strictly concave on $D_{e}\left(\mathbb{I}_{\Psi}\right)$.

Proof: Note that for each $f \in \overline{\mathcal{C}_{+}^{\mathbb{R}} \cap \mathcal{H}^{\infty}(\mathbb{D})}, \mathbb{I}_{\Psi}(f)<$ $\infty$ since $f \in \mathcal{H}^{\infty}(\mathbb{D})$. The strict concavity of $\mathbb{I}_{\Psi}$ on $\mathcal{C}_{+}^{\mathbb{R}} \cap$ $\mathcal{H}^{\infty}(\mathbb{D}) \subset D_{e}\left(\mathbb{I}_{\Psi}\right)$ follows from the strict concavity of the $\log$ function under the integral. Showing strict concavity on $D_{e}\left(\mathbb{I}_{\Psi}\right) \cap \partial\left(\mathcal{C}_{+}^{\mathbb{R}} \cap \mathcal{H}^{\infty}(\mathbb{D})\right)$ is also not difficult, we will do this for two cases. However, before continuing, keep in mind that for any $f \in D_{e}\left(\mathbb{I}_{\Psi}\right) \cap \partial\left(\mathcal{C}_{+}^{\mathbb{R}} \cap \mathcal{H}^{\infty}(\mathbb{D})\right)$, the set

$$
\left\{\theta \in[-\pi, \pi]: \Phi(f)\left(e^{i \theta}\right)=0\right\}
$$

has Lebesque measure zero, for otherwise the logarithmic integral will blow up to $-\infty$. Proceeding with our proof, we consider two possible cases separately:

Case I: $f_{1} \in D_{e}\left(\mathbb{I}_{\Psi}\right) \cap \partial\left(\mathcal{C}_{+}^{\mathbb{R}} \cap \mathcal{H}^{\infty}(\mathbb{D})\right)$ and $f_{2} \in \mathcal{C}_{+}^{\mathbb{R}} \cap$ $\mathcal{H}^{\infty}(\mathbb{D})$, and

Case II: $f_{1}, f_{2} \in D_{e}\left(\mathbb{I}_{\Psi}\right) \cap \partial\left(\mathcal{C}_{+}^{\mathbb{R}} \cap \mathcal{H}^{\infty}(\mathbb{D})\right)$. 
In both cases, it is straightforward to show that for all $0<$ $a_{1}, a_{2}<1$ satisfying $a_{1}+a_{2}=1$, and $f_{3}=a_{1} f_{1}+a_{2} f_{2}$ :

$$
\begin{aligned}
\log \Phi\left(f_{3}\right) & =\log \left[a_{1} \Phi\left(f_{1}\right)+a_{2} \Phi\left(f_{2}\right)\right] \\
& <a_{1} \log \Phi\left(f_{1}\right)+a_{2} \log \Phi\left(f_{2}\right) \text { a.e. on }[-\pi, \pi]
\end{aligned}
$$

From this it follows immediately that:

$$
\int_{-\pi}^{\pi} \Psi \log \Phi\left(f_{3}\right)<a_{1} \int_{-\pi}^{\pi} \Psi \log \Phi\left(f_{1}\right)+a_{2} \int_{-\pi}^{\pi} \Psi \log \Phi\left(f_{2}\right)
$$

and we may conclude that $\mathbb{I}_{\Psi}$ is strictly concave on $D_{e}\left(\mathbb{I}_{\Psi}\right)$.

Corollary 23: For a fixed $q \in Q^{-1}\left(\mathfrak{Q}_{+}(n, \mathbb{R})\right)$ and $\Psi \in \overline{\mathfrak{Q}_{+}(n, \mathbb{R})} \backslash\{0\}$, the Lagrangian $L_{q}: \overline{\mathcal{C}_{+}^{\mathbb{R}} \cap \mathcal{H}^{\infty}(\mathbb{D})} \rightarrow$ $\mathbb{R} \cup\{ \pm \infty\}$ defined by:

$$
L_{q}(f)=\mathbb{I}_{\Psi}(f)+\sum_{k=0}^{n} q_{k}\left(c_{k}-\frac{1}{2 \pi} \int_{-\pi}^{\pi} e^{i k \theta} \Phi(f)\left(e^{i \theta}\right) d \theta\right)
$$

is bounded from above on $\overline{\mathcal{C}_{+}^{\mathbb{R}} \cap \mathcal{H}^{\infty}(\mathbb{D})}$ and is strictly concave on $D_{e}\left(L_{q}\right)$. Furthermore, $D_{e}\left(L_{q}\right)=D_{e}\left(\mathbb{I}_{\Psi}\right)$.

Proof: We write the Lagrangian as

$$
L_{q}(f)=\frac{1}{2 \pi} \int_{-\pi}^{\pi} \Psi \log \Phi(f)+c^{\top} q-\frac{1}{2 \pi} \int_{-\pi}^{\pi} Q(q) \Phi(f)
$$

where $c=\operatorname{col}\left(c_{0}, c_{1}, \ldots, c_{n}\right)$ and $q=\operatorname{col}\left(q_{0}, q_{1}, \ldots, q_{n}\right)$. Since $Q(q) \in \mathfrak{Q}_{+}(n, \mathbb{R})$, we know that $\epsilon=\min _{\theta \in[-\pi, \pi]} Q(q)\left(e^{i \theta}\right)>0$. Also $M=\max _{\theta \in[-\pi, \pi]} \Psi\left(e^{i \theta}\right)<\infty$. Hence:

$$
L_{q}(f) \leq \frac{1}{2 \pi} M \int_{-\pi}^{\pi} \log \Phi(f)+c^{\top} q-\frac{1}{2 \pi} \epsilon \int_{-\pi}^{\pi} \Phi(f)
$$

Clearly, since the term $\int_{-\pi}^{\pi} \Phi(f)$ is nonnegative, the right hand side of (6) approaches $\infty$ only if $\int^{\pi} \log \Phi(f) \rightarrow \infty$. Now, for any sequence $\left\{f_{n}\right\} \subseteq \frac{-\pi}{\mathcal{C}_{+}^{\mathbb{R}} \cap \mathcal{H}^{\infty}(\mathbb{D})}$ such that

$$
\int_{-\pi}^{\pi} \log \Phi\left(f_{n}\right) \stackrel{n \rightarrow \infty}{\rightarrow} \infty
$$

it is trivial that

$$
\int_{-\pi}^{\pi} \Phi\left(f_{n}\right) \stackrel{n \rightarrow \infty}{\rightarrow} \infty
$$

and it is not difficult to check that $\frac{\int_{-\pi}^{\pi} \log \Phi\left(f_{n}\right)}{\int_{-\pi}^{\pi} \Phi\left(f_{n}\right)} \stackrel{n \rightarrow \infty}{\rightarrow} 0$. This implies that the right hand side of $(6)$ is bounded from above, hence so is $L_{q}$. Furthermore, since $L_{q}(f)=-\infty$ if and only $\mathbb{I}_{\Psi}(f)=-\infty$ we also have that $D_{e}\left(L_{q}\right)=$ $D_{e}\left(\mathbb{I}_{\Psi}\right)$. The strict concavity of $L_{q}$ on $D_{e}\left(L_{q}\right)$ then follows from the preceding lemma on the strict concavity of $\mathbb{I}_{\Psi}$ on $D_{e}\left(L_{q}\right)$ and from the linearity of $\frac{1}{2 \pi} \int_{-\pi}^{\pi} Q(q) \Phi(\cdot)$.

Since $L_{q}$ is a bounded from above and is a strictly concave functional on the convex set $D_{e}\left(L_{q}\right)$, if follows that if $L_{q}$ has a maximizer in $D_{e}\left(L_{q}\right) \subset \overline{\mathcal{C}_{+}^{\mathbb{R}} \cap \mathcal{H}^{\infty}(\mathbb{D})}$ then it is unique. The next theorem guarantees that a maximizer for $L_{q}$ always exists when $q \in Q^{-1}\left(\mathfrak{Q}_{+}(n, \mathbb{R})\right)$ :

Theorem 24: For any $q \in Q^{-1}\left(\mathfrak{Q}_{+}(n, \mathbb{R})\right)$, the maximum value of $L_{q}$ is attained at the rational element $g \in$ $\overline{\mathcal{C}_{+}^{\mathbb{R}} \cap \mathcal{H}^{\infty}(\mathbb{D})}$ given by: $g=\frac{a}{b}$ where $a, b$ are polynomials, $b b_{*}=Q(q)$ and $a b_{*}+a_{*} b=\Psi$. In particular, $g$ is the point at which the directional derivatives of $L_{q}$ vanish in any feasible direction relative to $D_{e}\left(L_{q}\right)$.

Corollary 25: For any $\Psi \in \overline{\mathfrak{Q}_{+}(n, \mathbb{R})} \backslash\{0\}$, the dual functional $\mathbb{J}_{\Psi}: Q^{-1}\left(\mathfrak{Q}_{+}(n, \mathbb{R})\right) \rightarrow \mathbb{R}$ defined by:

$$
\mathbb{J}_{\Psi}(q)=\sup _{f \in \mathcal{C}_{+}^{\mathbb{R}} \cap \mathcal{H}^{\infty}(\mathbb{D})} L_{q}(f)
$$

has the form:

$$
\mathbb{J}_{\Psi}(q)=c^{\top} q-\frac{1}{2 \pi} \int_{-\pi}^{\pi} \log Q(q)\left(e^{i \theta}\right) \Psi\left(e^{i \theta}\right) d \theta+K
$$

where $c=\operatorname{col}\left(c_{0}, c_{1}, \ldots, c_{n}\right), q=\operatorname{col}\left(q_{0}, q_{1}, \ldots, q_{n}\right)$ and $K=\frac{1}{2 \pi} \int_{-\pi}^{\pi} \Psi\left(e^{i \theta}\right) \log \Psi\left(e^{i \theta}\right) d \theta$ is a constant independent of $q$.

Proof: We have from the previous theorem that $\mathbb{J}_{\Psi}(q)=\sup _{f \in \mathcal{C}_{+}^{\mathbb{R}} \cap \mathcal{H}^{\infty}(\mathbb{D})} L_{q}(f)=\max _{f \in \mathcal{C}_{+}^{\mathbb{R}} \cap \mathcal{H}^{\infty}(\mathbb{D})} L_{q}(f)=L_{q}(g)$. All that remains is to plug in $\Phi(g)=\frac{\Psi}{Q(q)}$ in the definition of $L_{q}(g)$.

Note how the form of $\mathbb{J}_{\Psi}-K$ is the same for all $\Psi \in \overline{\mathfrak{Q}_{+}(n, \mathbb{R})} \backslash\{0\}$. It is also exactly the same form as the functional $\varphi$ that was given in $[5,(4.1)]$ for the case where $\Psi \in \mathfrak{Q}_{+}(n, \mathbb{R})$. It then follows by close inspection of the proofs that many results in [5] that are based on $\varphi$ having the form (7) modulo the constant $K$ actually holds true $\forall \Psi \in \overline{\mathfrak{Q}_{+}(n, \mathbb{R})} \backslash\{0\}$. In particular we have the following (with $\mathbb{J}_{\psi}$ replacing $\varphi$ ):

Lemma 26: [5, Lemma 4.2] The functional $\mathbb{J}_{\Psi}$ is finite and continuous at any $q \in \overline{Q^{-1}\left(\mathfrak{Q}_{+}(n, \mathbb{R})\right)}$, except at zero. The functional is infinite, but continuous, at $q=0$. Moreover, $\mathbb{J}_{\Psi}$ is a $C^{\infty}$ function in $Q^{-1}\left(\mathfrak{Q}_{+}(n, \mathbb{R})\right)$.

Remark 27: As a consequence of the previous lemma, we may extend the domain of definition of $\mathbb{J}_{\Psi}$ from $Q^{-1}\left(\mathfrak{Q}_{+}(n, \mathbb{R})\right)$ to $\overline{Q^{-1}\left(\mathfrak{Q}_{+}(n, \mathbb{R})\right)}$ and its range from $\mathbb{R}$ to $\mathbb{R} \cup\{\infty\}$. From this point on, we take the extended domain and range as the actual domain and range of $\mathbb{J}_{\Psi}$.

Lemma 28: [5, Lemma 4.3] The functional $\mathbb{J}_{\Psi}$ is strictly convex and defined on a closed, convex domain.

Proposition 29: [5, Proposition 4.6]For all $r \in \mathbb{R}$, $\mathbb{J}_{\psi}^{-1}(-\infty, r]$ is compact. Thus $\mathbb{J}_{\psi}$ is proper (i.e. $\mathbb{J}_{\psi}^{-1}(A)$ is compact whenever $A$ is compact) and bounded from below.

As a consequence of the preceding results, we have: 
Corollary 30: The functional $\mathbb{J}_{\Psi}$ has a unique minimum on $\overline{Q^{-1}\left(\mathfrak{Q}_{+}(n, \mathbb{R})\right)}$.

Theorem 31: If $q_{\text {min }} \in Q^{-1}\left(\mathfrak{Q}_{+}(n, \mathbb{R})\right)$ is a minimum for $\mathbb{J}_{\Psi}$ then the solution of the PRCEP is: $f=\frac{a}{b}$ where $b b_{*}=Q\left(q_{\min }\right)$ and $a b_{*}+b a_{*}=\Psi$. Conversely, suppose that $f=\frac{a}{b}$ is the solution to the PRCEP with $b$ being an antistable polynomial (i.e. having roots strictly in $\overline{\mathbb{D}}^{c}$ ) and $a b_{*}+b a_{*}=\Psi$. Then $q_{\min }=Q^{-1}\left(b b_{*}\right)$ is a unique minimum for $\mathbb{J}_{\Psi}$.

A straightforward approach to find or come close to the point which minimizes $\mathscr{J}_{\Psi}$ is to use a Newton gradient descent type algorithm which has been outlined in [3], [5], [8].

Example 32: Let the given partial covariance sequence be $\{0.2115,0.0728,-0.0396\}$. We choose the pseudopolynomial $\Psi(z)=z+2+z^{-1}$ which has two zeros on the unit circle, i.e. both at $z=-1$ and we seek a solution of the RCEP of degree 2. By using a Newton gradient descent algorithm we obtain the following value for $q_{\min }$ :

$$
q_{\min }=\operatorname{col}(8.6250,3.5000,2.0000)
$$

It can be checked that $q_{\mathrm{min}}$ is in the interior of $\overline{Q^{-1}\left(\mathfrak{Q}_{+}(n, \mathbb{R})\right)}$ and

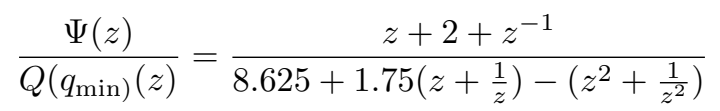

satisfies $\int_{-\pi}^{\pi} \frac{\Psi}{Q\left(q_{\min }\right)} e^{i k}=c_{k}$ for $k=0,1,2$. Finally, the associated solution of the PRCEP is

$$
f(z)=\frac{1}{\sqrt{8}} \frac{0.27935+0.31427 z+0.034919 z^{2}}{1+0.25 z-0.125 z^{2}}
$$

The remaining question is how do we compute the solution of a PRCEP if the minimum of $\mathbb{J}_{\Psi}$ lies on $\partial Q^{-1}\left(\mathfrak{Q}_{+}(n, \mathbb{R})\right)$ ? We suggest that if the solution is bounded then the approach of [5] should still be applicable. For this particular case, we assert that if $Q\left(q_{\text {min }}\right) \in$ $\partial \mathfrak{Q}_{+}(n, \mathbb{R})$ then all the roots of $Q\left(q_{\text {min }}\right)$ on $\partial \mathbb{D}$ will also be the roots of $\Psi$ on $\partial \mathbb{D}$. The following example suggests the validity of the above assertion.

Example 33: Consider the Carathèodory function

$$
f(z)=\frac{1}{2} \frac{1+\frac{z}{2}}{\left(1-\frac{z}{2}\right)}
$$

The positive covariance sequence associated with the spectral density $\Phi(f)$ is $\left\{1, \frac{1}{2}, \frac{1}{4}, \ldots\right\}$. We choose the pseudopolynomial $\Psi(z)=z+2+z^{-1}$ which has two zeros on the unit circle, i.e. both at $z=-1$, and we seek to find the solution of the RCEP of order $\leq 2$ corresponding to $\Psi$. By using a Newton gradient descent algorithm we obtain the following approximation for $q_{\min }$ :

$$
\hat{q}_{\min }=\operatorname{col}(2.0,0.66749,-1.3324)
$$

Examining $Q\left(\hat{q}_{\min }\right)$, then we find that its roots are: $\{2.0013,-1.0061,-0.99396,0.49967\}$. Note how two of the roots of $Q\left(\hat{q}_{\min }\right)$ are quite close to $z=-1$. Our claim is that if it were not for numerical discrepancies, these two roots would be exactly -1 and cancel the two corresponding roots of $\Psi$. Assuming that this is true, we find:

$$
\frac{\Psi(z)}{Q\left(\hat{q}_{\min }\right)(z)}=\frac{1.5001}{2.5010-\left(z+\frac{1}{z}\right)}
$$

which is the power spectral density of the Carathèodory function $\hat{f}(z)=0.49948 \frac{1+0.4997 z}{1-0.49967 z}$. It can be seen that $\hat{f}$ is close to the true function $f$ given in (8). Furthermore, by direct computation, the first three terms of the covariance sequence associated with $\frac{\Psi(\cdot)}{Q\left(\hat{q}_{\min }\right)(\cdot)}$ are:

$$
c_{0}=0.99889, c_{1}=0.49911, c_{2}=0.24939
$$

which is a reasonable approximation to the desired partial covariance sequence $\left\{1, \frac{1}{2}, \frac{1}{4}\right\}$. This example suggests that we may cancel out zeros of $Q\left(\hat{q}_{\min }\right)$ and boundary zeros of $\Psi$ which are very close to each other. If we do this, we obtain a solution which is close to the exact solution.

A better way of computation is the following. Since we assume that both roots of $\Psi$ are cancelled, we remove these roots from $\Psi$ to obtain the pseudoploynomial $\tilde{\Psi}=1$ (of degree zero). We now seek a solution of degree 2-1=1 since the same roots will also be cancelled in $Q\left(q_{\mathrm{min}}\right)$. Taking only the terms $c_{0}=1, c_{1}=\frac{1}{2}$ and disregarding $c_{2}$, we may easily solve the PRCEP for $\tilde{\Psi}=1$ since we know there will be no cancellation. Hence the minimizer of $\mathbb{J}_{\Psi}$ will be in the interior and the solution will be of degree 1. By applying a Newton gradient descent algorithm we find

$$
q_{\min }=\operatorname{col}(1.6667,-1.3333)
$$

and the solution $\tilde{f}=0.49996 \frac{1+0.49996 z}{1-0.49998 z}$ is close to the true solution given by (8). Later on we provide a general description of this second approach.

Remark 34: It should be noted that when the minimum of $\mathbb{J}_{\Psi}$ is close to or on the boundary, numerical problems may arise in computing the gradient and Hessian of $\mathbb{J}_{\Psi}$ when using a Newton gradient descent algorithm. However, we do not discuss this issue since it is outside the scope of this paper.

As it turns out, the generality of the observation in Example 33 can be formally proven. It is contained in the next theorem:

Theorem 35: The solution of the PRCEP is bounded if and only if $\mathbb{J}_{\Psi}$ has a stationary point in the interior or boundary of its domain. If $Q\left(q_{\text {min }}\right) \in \partial \mathfrak{Q}_{+}(n, \mathbb{R})$ and $q_{\text {min }}$ is stationary, then every root of $Q\left(q_{\min }\right)$ on $\partial \mathbb{D}$ will also be a root of $\Psi$ on $\partial \mathbb{D}$, and the solution of the PRCEP is of order less than $n$. In this case the solution is given by: $f=\frac{a}{b}$ where $b b_{*}=Q_{+}\left(q_{\min }\right), a b_{*}+b_{*} a=\tilde{\Psi}$, and

1) $Q_{+}\left(q_{\min }\right)$ denotes the symmetric polynomial that is left behind after all factors $\left(z^{ \pm 1}-e^{i \phi}\right)$ corresponding to the roots of $Q\left(q_{\min }\right)$ on $\partial \mathbb{D}$ have been removed from $Q\left(q_{\min }\right)$.

2) $\tilde{\Psi}$ denotes the symmetric polynomial that is left behind after all factors $\left(z^{ \pm 1}-e^{i \phi}\right)$ corresponding to 
the roots of $Q\left(q_{\min }\right)$ on $\partial \mathbb{D}$ have been removed from $\Psi$.

Based on Theorem 35 and a convergence theorem given below, we propose a computational procedure for solving the PRCEP, at least approximately, given a positive partial covariance sequence $c_{0}, c_{1}, \ldots, c_{n}$ and a pseudopolynomial $\Psi \in \overline{\mathfrak{Q}_{+}(m, \mathbb{R})}$ with $0 \leq m \leq n$. The procedure terminates in an exact or approximate bounded solution or a conclusion that the solution is not bounded.

Theorem 36: Let $\Psi \in \overline{\mathfrak{Q}_{+}(n, \mathbb{R})}$ and

$$
\begin{aligned}
q_{\text {min }} & =\underset{q \in Q^{-1}\left(\overline{\mathfrak{Q}_{+}(n, \mathbb{R})}\right)}{\arg \min } \mathbb{J}_{\Psi}(q) \\
q_{\text {min }, k} & =\underset{q \in Q^{-1}\left(\overline{\mathfrak{Q}_{+}(n, \mathbb{R})}\right)}{\arg \min } \mathbb{J}_{\Psi_{k}}(q)
\end{aligned}
$$

where $\left\{\Psi_{k}\right\}_{k>1} \subset \overline{\mathfrak{Q}_{+}(n, \mathbb{R})}$ is a sequence such that $\lim _{k \rightarrow \infty}\left\|\Psi-\Psi_{k}\right\|_{\infty}=0$. Then $\lim _{k \rightarrow \infty}\left\|q_{\min }-q_{\min , k}\right\|=0$ and $\lim _{k \rightarrow \infty}\left\|Q\left(q_{\min }\right)-Q\left(q_{\min , k}\right)\right\|_{\infty}^{\infty}=0$.

\section{Computational procedure:}

1) Choose a sequence $\left\{\Psi_{k}\right\}_{k \geq 1} \subset \mathcal{Q}_{+}(l, \mathbb{R}), 0 \leq l \leq$ $m$ converging strongly to $\Psi$ (note: no term in the sequence is at the boundary). This is straightforward to do in practice.

2) Check if there are roots of $\Psi$ on $\partial \mathbb{D}$ being approached closely by a sequence of roots of $\left\{Q\left(q_{k}\right)\right\}_{k \geq 1} \subset$ $\mathcal{Q}_{+}(n, \mathbb{R})$ as $k$ is increased.

3) Remove all boundary roots of $\Psi$ which are being approached (if any) and let the reduced order symmetric pseudopolynomial obtained from $\Psi$ after the removal of those roots be denoted by $\tilde{\Psi}$.

4) Count the number of roots removed, including multiplicities and with each conjugate pair being counted as one root. If the number is $r(r=0$ if there are no roots removed), use only the first n-r+1 terms $c_{0}, \ldots, c_{n-r}$ of the covariance sequence and ignore the rest.

5) Start at an interior point and execute a Newton gradient descent algorithm to check if a stationary point of $\mathbb{J}_{\tilde{\Psi}}$ exists. If the algorithm diverges (i.e. it tends towards a point on the boundary but the sequence of gradients does not tend to zero) then a stationary point does not exist and the solution is not bounded. If the algorithm converges, apply Theorem 31 and examine if the computed solution of order $n-r$ satisfies the original $n+1$ interpolation constraints exactly or approximately. In case the result is affirmative then the PRCEP is essentially solved, otherwise the solution of the PRCEP is not bounded.

We have shown part of the above procedure in action in the second part of Example 33. However, the above procedure does not guarantee an exact solution since it is numerically impossible to determine whether the limit of a sequence is truly at the boundary or is very close to it. Nonetheless, the solution obtained by this method will be close to the true solution in $L^{1}(\mathbb{D})$, the normed space of integrable functions on $\partial \mathbb{D}$.

\section{COnClusions}

Our main contributions in this paper are theoretical results on bounded solution of the PRCEP when $\Psi \in$ $\partial \mathfrak{Q}_{+}(n, \mathbb{R})$, i.e. the case where the chosen symmetric polynomial has zeros on the boundary. Furthermore, we propose a systematic method for computing the bounded solution, at least approximately, for this special case and provide two practical examples for illustration. The procedure also plays a dual role as a test for whether the associated solution is bounded or not.

Although we have only treated the PRCEP, it is very likely that the results here should also hold for the Nevanlinna-Pick interpolation with degree constraint as described in [8] and for the PRIP. They may also hold true in the case of matrix-valued interpolation. These are the generalizations we are currently pursuing. Finally, we mention that the theory of rational covariance extension seems to provide a framework for rational approximations of power spectral density functions. This idea is also currently being investigated.

\section{REFERENCES}

[1] T. T. Georgiou, "Realization of power spectra from partial covariance sequences," IEEE Trans. Acoust., Speech, Signal Processing, vol. 42, no. 8, pp. 1049-1070, 1987.

[2] C. I. Byrnes, A. Lindquist, S. V. Gusev, and A. S. Matveev, "A complete parametrization of all positive rational extensions of a covariance sequence," IEEE Trans. Automat. Contr., vol. 40, no. 11, pp. 1841-1857, 1995.

[3] C. I. Byrnes, S. V. Gusev, and A. Lindquist, "A convex optimization approach to the rational covariance extension problem," SIAM J. Contr. Optim., vol. 37, no. 1, pp. 211-229, 1998.

[4] T. T. Georgiou, "The interpolation problem with degree constraint," IEEE Trans. Automat. Contr., vol. 44, no. 3, pp. 631-635, 1999.

[5] C. I. Byrnes, S. V. Gusev, and A. Lindquist, "From finite covariance windows to modeling filters: a convex optimization approach," SIAM Rev., vol. 43, no. 4, pp. 645-675, 2001.

[6] A. Blomqvist, G. Fanizza, and R. Nagamune, "Computation of bounded degree Nevanlinna-Pick interpolants by solving nonlinear equations," Rep. no. 17, 2003, Mittag Leffler Preprint Series, Spring 2002/2003.

[7] A. Blomqvist, A. Lindquist, and R. Nagamune, "Matrix-valued Nevanlinna-Pick interpolation with complexity constraint: An optimization approach," IEEE Trans. Automat. Contr., vol. 48, no. 12, pp. 2172-2190, 2003.

[8] C. I. Byrnes, T. T. Georgiou, and A. Lindquist, "A generalized entropy criterion for Nevanlinna-Pick interpolation with degree constraint," IEEE Trans. Automat. Contr., vol. 46, no. 5, pp. 822-839, 2001.

[9] C. I. Byrnes, T. T. Georgiou, A. Lindquist, and A. Megretski, "Generalized interpolation in $H^{\infty}$ with a complexity constraint," Rep. no. 28, 2003, Mittag Leffler Preprint Series, Spring 2002/2003.

[10] H. I. Nurdin and A. Bagchi, "On the solutions of the rational covariance extension problem corresponding to pseudopolynomials having boundary zeros," 2004, submitted for publication.

[11] _ - "On the solutions of the rational covariance extension problem corresponding to pseudopolynomials having boundary zeros," Dept. App. Math., Univ. of Twente, Memorandum (preprint) 1716, March 2004, ISSN 0169-2690. [Online]. Available: http://www.math.utwente.nl/publications/publ2004.html

[12] R. T. Rockafellar, Conjugate Duality and Optimization, ser. Regional Conference Series in Applied Mathematics. SIAM, 1974, no. 16. 